\title{
Desarrollo de las nuevas tecnologías de información en México
}

María de la Luz Casas Pérez

El PRESENTE TRABajo eXPLORA el desarrollo de las nuevas tecnologías experimentado por México fundamentalmente durante el presente siglo. Se entiende aquí por nueva tecnología toda aquella herramienta de información y comunicación que resulta novedosa en su momento, y que viene a representar un cambio económico y cultural en la sociedad de la época. Partimos de la base, de que la introducción de nuevas tecnologías de información y de comunicación a través de la historia, se ha asociado siempre a importantes transformaciones estructurales de los sistemas socio-económicos, políticos y culturales correspondientes. ${ }^{2}$ Desde ese punto de vista, las nuevas tecnologías son todas aquellas que con la telegrafia y la telefonía, rompieron las barreras del tiempo y del espacio para propiciar un despegue de las actividades comerciales y culturales de la época. El telégrafo es el gran iniciador de la modernidad del siglo xx, a él le siguen la telefonía, la radio y la televisión. Hoy por hoy, la computación, el internet y toda una serie de tecnologías derivadas de las anteriores, representan las nuevas herramientas para la comunicación y el intercambio de bienes, servicios, información y entretenimiento.

En México, la introducción de cada una de estas tecnologías significó poderosos avances en el desarrollo del comercio y de la sociedad. Como veremos más adelante, la adopción de estas tecnologías fue vista primeramente como una actividad dependiente del Estado, y posteriormente éste fue replegándose al punto de dejar estas actividades preponderantes en manos de la iniciativa privada. He aquí algunos de los datos más importantes en relación a esta evolución y desarrollo.

1. Instituto Tecnológico de Estudios Superiores de Monterrey, Campus Morelos.

2. Esta idea fue planteada por primera vez por el economista canadiense Harold Innis, en su famosísima obra Communication and Empire (Oxford, 1950). 


\section{Los primeros desarrollos tecnológicos}

El desarrollo de las tecnologías de información arranca en México con la implantación del servicio telegráfico en México (1849-1851), que para la década de 1870 contaba ya con $8,000 \mathrm{~km}$ de longitud. Alrededor de 1880 se hicieron las primeras pruebas telefónicas y poco después se instaló la primera red telefónica en la Ciudad de México. En 1883 se realizó la primera conferencia telefónica internacional de México (Matamoros-Brownsville); hacia 1890 la red telefónica contaba ya con 1100 suscriptores (un aparato por cada 10,000 habitantes). ${ }^{3}$

Durante el Porfiriato ${ }^{4}$ hubo un gran avance en las comunicaciones y los transportes. La Revolución Mexicana (1910-1917) implicó un retroceso para las comunicaciones, por lo que fue hasta 1924 que se inauguró la primera central telefónica automática en México. ${ }^{5}$

Hacia 1930 había en México apenas medio teléfono por cada cien habitantes; en 1950 la cifra se había duplicado, y en 1970 pasaba ya de tres teléfonos por cada cien habitantes. En 1985 la cifra se incrementó casi a nueve teléfonos por cada cien habitantes. La mayor parte de la infraestructura telefónica instalada hasta 1983 había sido instalada entre 1968 y 1982.

En cuanto al número de llamadas telefónicas por habitante, ésta era de treinta en 1965 y creció a 153 en 1980.

Por lo que toca a la comunicación punto-multipunto, es decir un emisor y muchos receptores, en 1928 se realizó la primera transmisión de radio en México, aunque las primeras estaciones comerciales de radio con programación regular, no se inauguraron sino hasta 1935. La primera estación experimental de televisión se instaló en 1947 y la televisión comercial se inauguró en 1950.

\section{Un primer momento: las tecnologías educativas}

En sus primeros momentos, la radio y la televisión econtraron un buen nicho en la actividad comercial dirigida al entretenimiento. Tuvieron que pasar varias décadas, antes de que el Estado hiciese conciencia de que la gran infraestructura podría ser utilizada para otros fines, particularmente los educativos.

3. Federico Kuhlmann; Antonio Alonso C. y Alfredo Mateos. Comunicaciones: Pasado y Futuros. Fondo de Cultura Económica, México, 1989, pp. 90-91.

4. Régimen del Presidente Porfirio Díaz (1877-1880 y 1884-1911).

5. Mientras que en los países desarrollados, semejante servicio se habia ocurrido por lo menos diez años antes. 
Teniendo como tenía, una gran responsabilidad en el uso estratégico de las comunicaciones, el Estado mexicano inició las tareas para desarrollar una infraestructura de comunicación que diera sustento a otras necesidades, entre ellas la de la difusión de la cultura.

La experiencia mexicana de Televisión Educativa toma cuerpo a partir de 1965, fecha en que la Dirección General de Educación Audiovisual de la Secretaría de Educación Pública inicia un extenso plan, coherente y sistemático, de utilización de la televisión para ponerla al servicio de la alfabetización. Convencidos de la eficacia educativa de los medios, se puso énfasis en el hallazgo de un método válido y eficaz para alfabetizar. Un equipo de maestros pedagogos estudió las posibilidades, iniciando las emisiones en circuito abierto a través de una red de emisoras de televisión en todo el país.

El esfuerzo alfabetizador original animó a las autoridades a emprender un programa semejante de educación media superior (telesecundaria), que ofrecía la posibilidad de cursar las materias de la escuela media básica a todos los que no hubieran podio inscribirse por falta de cupo, a los que vivieran en zonas apartadas o en regiones cuya reducida población escolar no justificara la construcción y mantenimiento de un plantel de enseñanza secundaria y en general, a quienes por otras circunstancias no hubieran podido efectuar otros que los de la escuela básica. Este sistema quedó totalmente integrado en 1969. El sistema de transmisión, sin embargo, era todavía abierto a través de estaciones repetidoras localizadas en distintas partes de la República mexicana. ${ }^{6}$

Este fue el primer intento de utilizar una nueva tecnología de transmisión de información y de cultura con otros fines fuera de los comerciales. Era evidente que el gobierno mexicano estaba reconociendo la tremenda necesidad de comenzar a utilizar la tecnología, especialmente una tan poderosa como lo era la tecnología audiovisual a través de la televisión con fines propiamente educativos. Otros intentos sobrevinieron posteriormente, la creación de Radio Educación, TV UNAM, y el Instituto Latinoamericano de la Comunicación Educativa (ILCE), significaron para México una oportunidad de oro para comenzar a poner a las nuevas tecnologías al servicio de la sociedad.

No obstante que, desde la década de los años cincuenta y sesenta, instituciones como la Universidad Nacional Autónoma de México habian hecho ya esfuerzos por utilizar las tecnologías de comunicación para la enseñanza, fue en la década de los setenta que se propició un importante desarrollo institucional, permitiendo así abrir la oferta educativa a nuevas posibilidades tecnológicas. Así por ejemplo la televisión

6. Jesús García Jiménez. Televisión educativa para América Latina. Pornua. México, 1970, p.p. 255-259. 
universitaria se hace presente con series de difusión cultural y de formación profesional y se elaboran importantes modelos de adecuación de los contenidos académicos a los lenguajes televisivos. ${ }^{7}$

Las décadas de los años setenta y ochenta fueron instrumentales para lograr desarrollar la infraestructura tecnológica y a toda una planta de profesionales dedicados a la enseñanza a través de las tecnologías de educación a distancia. Lamentablemente, los esfuerzos se vieron mermados por las subsecuentes crisis económicas que vivió el país $(1976,1982$, y 1985) y que hicieron mella no solamente en la política económica del país, sino también en toda su estructura financiera, social y de supervivencia.

En fechas más recientes, se ha aprovechado la capacidad instalada del país, para crear una red satelital para la educación, llamada Edusat que es un sistema de transmisión y recepción de señal televisiva que inició sus actividades en diciembre de 1995. La programación de Edusat se transmite por seis canales de televisión propios de la Red, por medio de los satélites Solidaridad I y Morelos II, por frecuencias de UHF, a través de los sistemas comerciales de cable y la televisión pública.

Durante 1996 y el primer semestre de 1997, los esfuerzos de la Red Edusat se han orientado de manera prioritaria a dotar a los planteles educativos de la infraestructura requerida para la recepción de su señal. También se ha avanzado en la tarea de desarrollar el perfil de la programación y los apoyos adicionales que favorecen el mejor aprovechamiento de los materiales que se transmiten. Para el primer semestre de 1997, se habían instalado más de 15000 equipos receptores. ${ }^{8}$ No obstante, y pese a los esfuerzos del Estado por utilizar la tecnología instalada con fines educativos, el énfasis del desarrollo tecnológico sigue estando en el sector comercial.

El final de los años ochenta y el principio de los años noventa vieron el nacimiento y la apertura al uso de nuevas tecnologías. Las instituciones educativas, entre ellas la Universidad Nacional Autónoma de México y el Instituto Tecnológico y de Estudios Superiores de Monterrey, fueron las primeras instancias en abrir sus portales de salida a Internet. Este desarrollo permitió que la planta académica y estudiantil se incorporaran a la tecnología como apoyo a sus actividades.

Los pasos que daría México durante esos años serían fundamentales en la evolución de la infraestructura de telecomunicaciones que daría paso a la instalación en

7. Patricia Fernández Hernández. "Experiencias institucionales de educación a distancia". Ponencia presentada en ocasión del iv Encuentro-Taller Internacional de Educación Continua del Programa Columbus, septiembre de 1988. P. 4.

8. Nieto, Dulce. Perfil de la educación en México. Secretaría de Educación Pública, http://www.sep.gob.mx/ sen/perfil/p7_1.htm 
ese país de nuevas tecnologías apropiadas por la iniciativa privada y auspiciadas por el Estado con diversos fines. En sus inicios, las nuevas tecnologías fueron aprovechadas dentro del marco de las instituciones de educación permitiendo el trabajo en red, la colaboración institucional y el intercambio de ideas fueron transformando otros ámbitos de la vida social, al punto de incidir claramente en la integración de organismos comerciales y de servicios que poco a poco invadieron los ámbitos de la vida cotidiana y el comercio.

\section{Un segundo momento: la instalación de la infraestructura tecnológica}

En gran medida, gran parte de la capacidad instalada en las industrias de la computación y la electrónica, dependieron de la importación de productos manufacturados y tecnología, bienes de capital e insumos básicos para la industria. Por tal motivo, México experimentó un fuerte atraso en el desarrollo de una infraestructura de comunicaciones y nuevas tecnologías, que todavía sigue padeciendo. Por otra parte, la incapacidad tecnológica para producir productos manufacturados en esta industria, también relegó a nuestro país respecto de otros modelos de desarrollo y del proceso de producción y competitividad de los bienes y servicios de alto valor agregado con los países industrializados.

En cuanto a la infraestructura de comunicaciones que México tuvo que desarrollar para poder, eventualmente, vincularse con el exterior, destaca la comunicación vía satélite. Al respecto, podemos argumentar, que si bien ésta comenzó oficialemente desde 1966, año en que México, junto con Chile y Panamá ingresaron formalmente a la Organización Internacional de Comunicaciones por Satélite (INTELSAT), haciendo con ello posible el establecimiento provisional aplicable a un sistema comercial internacional de telecomunicaciones por satélite, no fue sino hasta 1971 que quedaron oficialmente aprobados los acuerdos relativos a la operación del sistema. Ya en 1968, con motivo de la xix Olimpiadas, México había comenzado a utilizar el satélite experimental ATS-3, propiedad de la NASA y rentado a INTELSAT. Al año siguiente, en 1969, México establecía una conexión internacional permanente a través del satélite INTELSAT III, situado sobre el Océano Atlántico. ${ }^{9}$ Posteriormente vendrían los satéli-

9. Ligia María Fadul, Fátima Fernández y Héctor Schmucler, "Satélites de Comunicación en México", en Ruth Gall, et al., Las actividades espaciales en México: Un revisión critica, Fondo de Cultura Económica/SEp/CONACYT, 1986, p. 123. 
tes de la serie INTELSAT IV-A e INTELSAT V cubriendo esencialmente el Océano Altántico. Tiempo después, otra serie de satélites, los WESTAR de la serie III y IV, así como los Galaxy dieron cobertura durante los siguientes años al territorio nacional. A raíz de esta serie de lanzamientos, tuvo lugar una auténtica revolución en la difusión y retransmisión de la información a distancia. Aunque en primer término los satélites fueron utilizados con fines militares o comerciales, llegó el momento en que la posibilidad técnica se abrió a los particulares.

En la década de los años ochenta, México decidió poner en órbita un sistema de satélites artificiales de comunicación, cuyo nombre sería "Señor de los cielos" (Ilhuicahua). La serie estaria constituida por tres satélites, uno para servicio permanente, otro para casos de emergencia y un tercero que permanecería en tierra como reserva. A partir de 1983 la serie de satélites recibió un nuevo nombre: Sistema de Satélites Morelos.

Podemos decir que, a partir de la década de los años ochenta comenzó en México la difusión directa de señales de satélite. Aunque ya desde décadas anteriores, el Estado se había hecho cargo de la utilización del espacio aéreo con distintos fines. Lo anterior, haciendo valer del precepto constitucional establecido en el Artículo 42, fracción Vl que indica que el espacio situado sobre el territorio nacional es parte integrante del mismo, con la extensión y modalidades establecidas por el derecho internacional. Por otra parte, tal y como lo establecía el Artículo 28 constitucional previa a su modificación, el Estado ejercería de manera exclusiva en las áreas estratégicas, entre las que se encontraba la comunicación vía satélite, las actividades que expresamente señalen las leyes, y que expida el Congreso de la Unión. Desde ese momento, sin embargo, se sentaron las bases para la futura utilización de la infraestructura satelital, ya que en 1981 un decreto disponia que la Secretaría de Comunicaciones y Transportes podría intervenir en la instalación y operación de satélites y subsistemas asociados y, posteriormente, en 1994 finalmente se abrograba parte del precepto constitucional, para indicar que si bien la actividad de la comunicación vía satélite seguía siendo estratégica para la nación, su instalación y operación ya no sería competencia exclusiva del Estado mexicano.

Otro momento importante en el desarrollo del sector de las telecomunicaciones, lo constituye la privatización de la industria telefónica.

La urgente modernización y expansión de las telecomunicaciones nacionales requería de grandes inversiones, pero para el Gobierno Federal era imposible distraer los recursos fiscales necesarios para atender las demandas de salud, educación, vivienda y adecuación del resto de la infraestructura. Por lo que el Estado, ejerciendo la rectoría en las telecomunicaciones, indujo su desarrollo mediante un 
nuevo marco regulatorio que permitía la expansión de las mismas, iniciando con la privatización de Teléfonos de México, que ha alcanzado los 10 millones de líneas. Lo cual, aunado a los grandes avances tecnológicos y a los novedosos productos y servicios la convierten en la Empresa de Telecomunicaciones más grande e importante de América Latina.

1989 representó el inicio de una nueva etapa de desarrollo tecnológico, financiero y de servicios cuyos objetivos eran el mejoramiento de la calidad del servicio, el crecimiento y la expansión del mismo, la modernización tecnológica y la diversificación de los bienes y servicios. Como una respuesta a este reto de modernización, y para competir en la venta y promoción de los servicios digitales, Teléfonos de México inició las operaciones del Centro de Telecomunicaciones Avanzadas, institución de investigación integrada por un grupo de especialistas altamente calificado en la materia. Este centro contaba con la primera Red Digital de Servicios Integrados (RDSI) y fue constituido para funcionar como un laboratorio cuyo objetivo era desarrollar nuevos servicios. En ese año, Teléfonos de México inició su participación en el mercado de los servicios celulares a través de su filial Radiomóvil Dipsa, S. A. de C. V., para satisfacer eficazmente la demanda de los usuarios que requieren telecomunicaciones personales y tecnología de vanguardia. En tanto, el Centro de Investigación y Desarrollo logró la aplicación de tecnología avanzada para el mejoramiento de la red nacional, diseñó sistemas y desarrolló equipos complementarios que han hecho más eficiente la operación de los ya existentes. A fin de modernizar su actividad interna Teléfonos de México inició un programa de automatización para los procesos básicos de atención al usuario, tales como la recepción de solicitudes de líneas y servicios, quejas y aclaraciones, reparación, instalación y cobranza. El presidente Salinas en su "Plan Nacional de Desarrollo 1989-1994" en lo referente a la modernización de las telecomunicaciones señalaba: "La indispensable modernización y expansión de las telecomunicaciones requerirá de grandes inversiones, que deberán financiarse con participación de los particulares; el propósito es no distraer recursos fiscales necesarios para atender las legítimas demandas de salud, educación, vivienda y adecuación del resto de la infraestructura. El Estado ejercerá la rectoría en las telecomunicaciones e inducirá su desarrollo, mediante un nuevo marco regulador que tenga en cuenta el cambio tecnológico habido en los últimos años. La regulación dará la debida seguridad jurídica a los participantes en el sector".

En septiembre de 1989, el gobierno federal anunció su intención de privatizar Teléfonos de México, vendiendo su participación en el capital de la empresa y facilitando así el proceso de modernización de las telecomunicaciones en nuestro país. Pues el desarrollo tecnológico modificó las condiciones tradicionales de organiza- 
ción existentes en el sector y varios países cuyas telecomunicaciones estaban bajo un régimen de propiedad estatal, procedieron a abrirlas a la competencia y a encomendar la parte medular de ellas al sector privado. ${ }^{10} \mathrm{El}$ sector privado tendría en su haber varios años para actualizarse y eficientar una estructura burocrática y deficiente y actualizar tecnológicamente una infraestructura de interconexión que permitiria, finalmente a nuestro país, hacer frente a los compromisos contraídos para la futura apertura de los mercados mundiales.

1994 coincide, por supuesto, con la puesta en marcha del Tratado de Libre Comercio para América del Norte, que descansa entre otras cosas, en un rápido desarollo de la infraestructura de telecomunicaciones. Era obvio, por ejemplo, que México tendría que lograr un gran avance en el campo de la informática y las telecomunicaciones si quería entrar en un convenio comercial con las dos más grandes potencias económicas de América del Norte.

Desde ese momento, se hizo evidente que la informática y las telecomunicaciones, serían la nueva piedra angular para el crecimiento de las sociedades. Dos campos que habían avanzado por separado, a partir de la mitad de la década de los años ochenta se encuentran para producir la naciente telemática, es decir, la combinación de telecomunicaciones e informática. Con la conjunción de ambos procesos se logra, por primera vez en la historia de la humanidad, la abolición de las limitantes del tiempo y el espacio. La informática produce sistemas de procesamiento de información cada vez más rápidos y con mayor capacidad de almacenaje y procesamiento, mientras que, por otra parte, las telecomunicaciones avanzan eliminando las distancias. Así queda garantizada la rapidez del proceso informativo y se asegura la instantaneidad de las transmisiones.

La incidencia reciente de la telemática en los procesos productivos es no sólo evidente, sino incluso impactante. La informática, relacionada con servicios de consulta a bancos de datos representa una actividad creciente en la economía mexicana.

Se calcula que en 1964 había en México 65 instalaciones de cómputo, tomando en consideración tanto el sector público como el sector privado. ${ }^{11}$ No obstante el crecimiento de la industria electrónica, en un inicio el mayor usuario del procesamiento automatizado de la información fue el Estado Mexicano. Sólo durante el período 1979-1982, la demanda de bienes y servicios informáticos se vio rebasada por las compras que realizó el sector privado. Todas las evidencias actuales indican

10. Teléfonos de México, "Origenes de la Telefonia", en http://www.telmex.com.mx/estoes/historia/ historia_frame.htm

11. Secretaría de Hacienda y Crédito Público de México, "Programa de Desarrollo Informático", INEGI, México, 1980. 
que las industrias de información no sólo son estratégicas por la importancia de la automatización de los procesos informativos, sino que constituyen el corazón mismo de los nuevos sistemas productivos en gestación y en desarrollo. Así, la industria electrónica en su conjunto observó una tasa de crecimiento de su producto del 10.9 por ciento durante la década de los setenta, muy superior al promedio de 7.1 por ciento de las industrias de transformación y al 7.0 del PIB. ${ }^{12}$ Para 1982 México importaba en su mayor parte una gran cantidad de componentes para equipos de cómputo; sin embargo, la apertura de mercados influyó notablemente tanto en la proliferación de importaciones, como en la producción nacional asistida por inversión extranjera.

Los usos de las nuevas tecnologías de información varían, como hemos visto, desde el sistema informático nacional (bases de datos y sector público) hasta el comercio electrónico, pasando por el sistema bancario.

La importación de equipos electrónicos se ha liberalizado en muchos países latinoamericanos, y por lo tanto, el material informático está ampliamente disponible. Los precios de los equipos no son muy diferentes a los de Norteamérica. Las mejoras en la infraestructura de telecomunicaciones, especialmente en las áreas urbanas, y la disminución de los costos de conexión a Internet están dando por resultado índices de crecimiento muy altos de conectividad. Por ejemplo, en México el número de sitios registrados aumentó un 350 por ciento entre octubre de 1996 y octubre de 1997, y en su momento se pronosticaba una población de un millón de usuarios de Internet para $1998 .^{13}$

Para 1996, el Internet Domain Survey registraba ya 13,787 servidores de internet en México. ${ }^{14}$ Para abril de 1999 Nua Internet Surveys reportó que había 500,000 usuarios accesando internet en México. Esta cifra está basada en una encuesta que encontró que en abril de 1999 existían 1 millón 400,000 computadoras pertenecientes a las clases media alta y alta en México, con la posibilidad de que 280,500 probablemente se agregaran al final de ese mismo año. Si tomamos en consideración que para cada una de ellas, quizás haya un promedio de dos personas usando cada computadora, podriamos calcular un promedio de 504,900 usuarios. Sin desestimar al usuario doméstico, que se calcula en 99,200 cuentas de acceso, y al usuario proveniente de instituciones educativas en alrededor de 22,500 , es posible detectar

12. Noriega, Carlos. "Industria electrónica, instrumento para el desarrollo", México, 1982. p 13.

13. Santoyo, Ramón V., "Internet, Banca, y Comercio Electrónico", Grupo Telelink, http:// www.telelink.com.mx/docs/tecnofin/sld001.htm, 1997; citado en Charles Davis, "Algunos desafios para el desarrollo del comercio electrónico en la América Latina de habla hispana" en: http:// ute.edu.ec/ mjativa/ce/tendencias.html, marzo 1998 .

14. Fuente: Internet Domain Survey (hhtp://www.nw.com), 1996. 
que la mayoría de las conexiones a internet provienen de alrededor de 153,150 empresas, entre las cuales se encuentran, por supuesto instituciones grandes y medianas que han entrado a la dinámica de la economía global. ${ }^{15}$

\section{Un tercer momento: la apertura de la infraestructura de telecomunicaciones y nuevas tecnologías}

El rápido desarrollo de la infraestructura de telecomunicaciones, especialmente en los renglones de telefonía y comunicación vía satélite, así como el desarrollo de la industria informática, ha propiciado que distintos actores dentro de la vida económica, política y social de México hayan entrado a la adopción de nuevas tecnologías de comunicación como formas de desarrollo acelerado de ciertos sectores.

Los compromisos signados por México en el marco de los acuerdos del Tratado de Libre Comercio de América del Norte, han propiciado que no solamente compañías mexicanas, sino también norteamericanas y canadienses hayan ayudado al

Gastos en comercio electrónico en América Latina

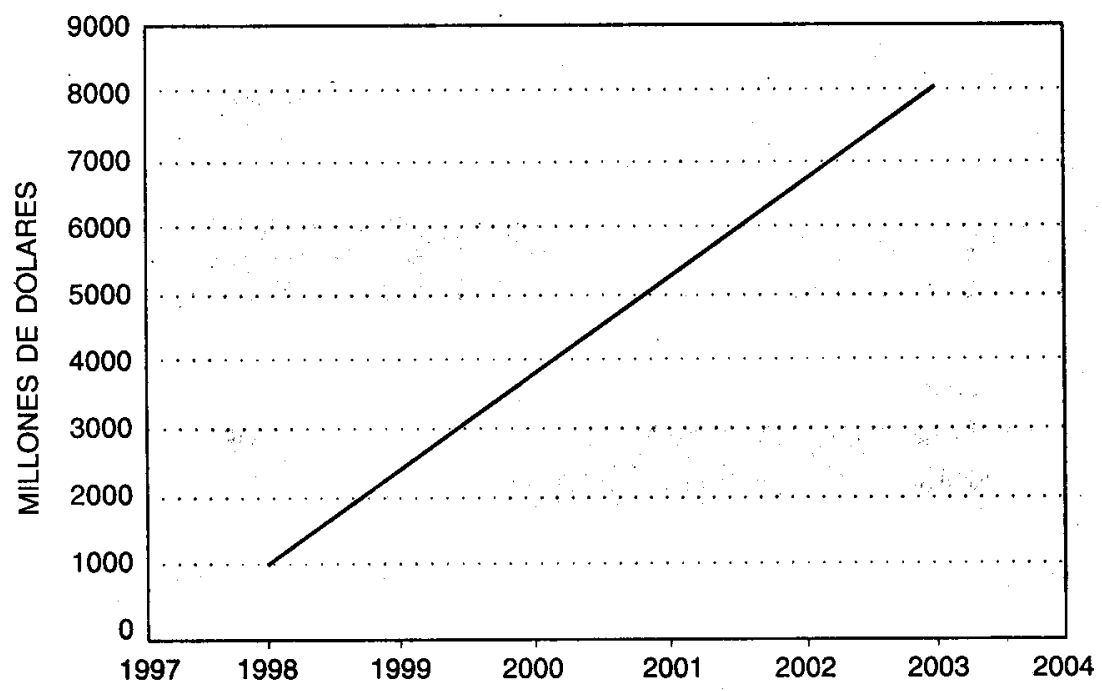

Fuente: International Data Corporation, Junio de 1999, http:// www.headcount.com/globalsource/ecommerce/index.htm?choice=Latin+America

15. Fuente: http://www.headcount.com/count/datafind.htm?id=153\&choice $=$ country 
gran impulso en el sector de las telecomunicaciones y la informática en México. La industria norteamericana y canadiense reaccionó ante las nuevas economías de bloque fusionándose en grandes conglomerados. Tanto las firmas norteamericanas como las canadienses han implementado tecnologías sobre territorio mexicano, ayudando a las compañías mexicanas a desarrollar tecnologías celulares, microondas y fibra óptica con el propósito de alcanzar una mayor competitividad en el comercio transfronterizo y ayudar a las compañías norteamericanas y canadienses a penetrar los mercados mexicanos con mayor eficiencia.

La incrementada presencia de proveedores de servicios de internet, por ejemplo, es una muestra de la creciente demanda del servicio y de los nuevos usos de la información a través de las nuevas tecnologías. La popularidad alcanzada por el comercio electrónico en los últimos años, es otro indicador de la tendencia de los distintos actores económicos involucrados en el proceso de aprovechamiento de las tecnologías de información.

De acuerdo con datos obtenidos por International Data Corporation (IDC), el gasto registrado por comercio electrónico en Latinoamérica durante 1998 se incrementó en un 361 por ciento con respecto de 1997, llegando a un total de 167 millones de dólares, y se espera que alcance los 8 billones de dólares para el año 2003. ${ }^{16}$

Lo anterior se deriva del hecho de que los consumidores están incorporándose rápidamente al uso de esta tecnología para la adquisición de información, bienes y servicios, y que la mayoría de sus empresas ha comenzado a producir soluciones informáticas integrales para la atención a sus clientes.

Ahora bien, si tomamos en consideración la proporción de usuarios registrada en los tres países del bloque económico de América del Norte, bajo el paraguas del TLC, es posible observar que los Estados Unidos cuentan actualmente con un promedio de 92 millones de usuarios mayores de 16 años de edad. El número de usuarios en Estados Unidos y Canadá se incrementó 16 por ciento en sólo nueve meses, sin embargo el número de consumidores en línea creció un 40 por ciento hasta llegar a 28 millones durante el mismo período. ${ }^{17}$

Para Canadá, la cifra que reporta Nua Internet Surveys es de 13 millones 500,000 usuarios para marzo de $1999,{ }^{18}$ que comparados con los 500,000 usuarios mexicanos que reporta la misma empresa, indican que el mercado aunque poco desarrolla-

16. Fuente: International Data Corporation, Junio de 1999 http://www.headcount.com/globalsource/ ecommerce/index.htm?choice=Latin+America

17. Fuente: http://www.headcount.com/count/datafind.htm?choice $=$ country\&choicev[] $=$ The+US

18. Fuente: http://www.headcount.com/count/datafind.htm? choice=country\&choicev[] $=$ Canada 
do en nuestro país, sufrirá un incremento exponencial en los próximos años, simplemente por la cercanía y por los compromisos adquiridos con sus vecinos del Norte. ${ }^{19}$

\section{Usuarios de Internet en millones}

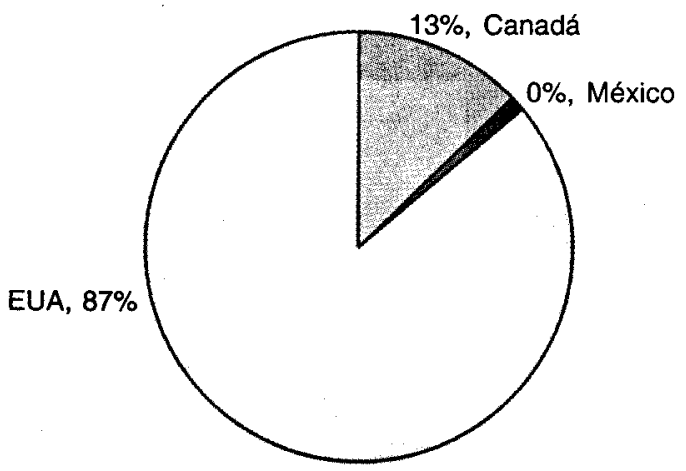

Fuente: Nua Internet Surveys, 1999 http://www.headcount.com/count/ datafind.htm?choice $=$ country \&choicev

Ahora bien, el crecimiento del sector viene acompañado de una fuerte inversión en la industria de las telecomunicaciones. Desde la privatización de Telmex en 1990, se dió inicio un fuerte proceso de integración de los servicios, no sólo en telefonía, sino en servicios de valor agregado dependiente de las telecomunicaciones. La ampliación de servicios de telefonía a telefonía celular, telefonía de larga distancia, conexión a internet y otros servicios de valor agregado, han diversificado la industria de las telecomunicaciones y la han acercado a la industria electrónica y de la informática.

La relación iniciada por México dentro del marco del TLc ha obligado, además, a hacer los servicios de interconexión posibles, de manera que grandes compañías trasnacionales como AT\&T y MCI WorldCom han invertido una buena cantidad de miles de millones de dólares en los mercados de larga distancia en México.

No obstante, e indepenientemente de que el sector ha experimentado una fuerte interconexión en interdependencia con los servicios telefónicos norteamericanos, los Estados Unidos acusaron a Telmex a través de la Organización Mundial de Comercio de incurrir en prácticas monopólicas, ejerciendo una importante presión sobre nuestro país y sobre la COFETEL para que el gobierno mexicano instrumentara una reglamentación mucho más rígida sobre la empresa telefónica mexicana.

19. Fuente: http://www.headcount.com/count/datafind.htm?choice=country\&choicev[]=Mexico 
La Comisión Federal de Competencia detectó prácticas monopólicas en cinco mercados relevantes que son: larga distancia nacional e internacional, telefonía local, tráfico interurbano y acceso. Estas prácticas son inadmisibles si México desea seguirse preciando de ser un país en el cual reine la apertura informativa y comercial, sobre todo por su pertenencia a la organización Regulatel, en la que participan países como Argentina, Bolivia, Brasil, Colombia, Costa Rica, Cuba, Ecuador, El Salvador, Guatemala, Honduras, Nicaragua, Panamá y Venezuela.

Lo anterior le dará un poco más de holgura a la empresa mexicana en sus operaciones en el extranjero, especialmente en los Estados Unidos en donde el mercado ya estaba reaccionando a la intervención tan feroz de la empresa mexicana. Telmex había estado incursionando ya en los mercados estadounidenses, ofreciendo servicios de telefonía a poblaciones vecinas en Texas y Arizona, con el propósito de obtener parte de un mercado cuyo valor anual es de más de dos mil millones de dólares.

Dentro del mercado de telefonía inalámbrica, Iusacell en su relación con Bell Atlantic, es una de las compañías competidoras más fuertes de Telmex, con planes para vincularse a su vez con empresas trasnacionales de la importancia de Lucent Technologies, sin embargo, el liderazgo en infraestructura instalada, sigue siendo de Telmex. ${ }^{20}$

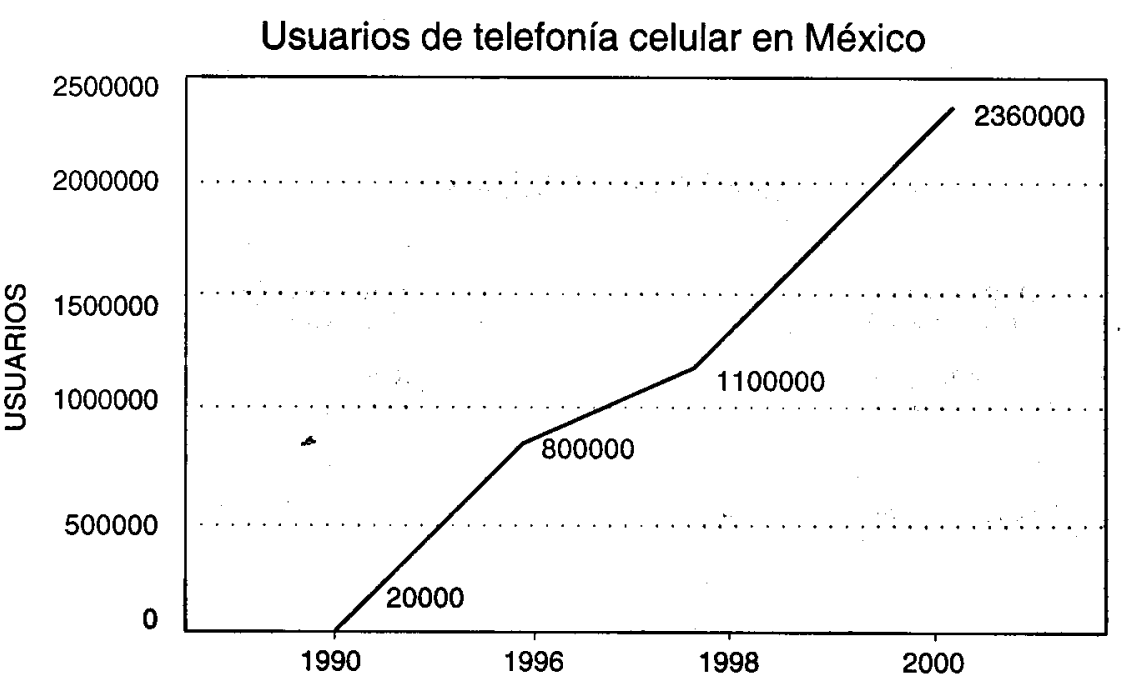

Fuente: IDC, 1999, http://www.headcount.com/globalsource/ecommerce/index.htm

20. Aproximadamente 3 millones 250 mil mexicanos tienen teléfono celular. El mercado está experimentando una penetración del 3.4 por ciento anual. Se espera que alcance 10 a 12 por ciento para el año 2003. En este momento, se espera que la industria invierta alrededor de 3 mil 400 millones de dólares en el lapso de los próximos cinco años. Norman Lerner. "A bright Market Matures", en Telecommunications on line. http://www.telecoms-mag.com/issues/199910/tci/mexico.html. 
Aún cuando la COFETEL ha autorizado la incursión de otras compañías como Unefón y Midicel dentro del mercado de la telefonía, especialmente inalámbrica, para estas compañías todavía es necesario negociar acuerdos de interconexión con la infraestructura de Telmex, por lo que es de suponerse que el mercado seguirá estando en sus manos por un buen tiempo. ${ }^{21}$

Las presiones del mercado se han dejado sentir en varios frentes. Uno de ellos es el de la telefonía móvil. Telmex reaccionó separando su división móvil de telefonía y las subsidiarias internacionales de Telmex para crear una nueva empresa llamada América Móvil, lo cual le permitirá relevar a su empresa de las presiones regulatorias. Ello le dio también un espacio a la cOFETEL para generar un esquema nuevo y diferente de reglamentación conocido como regulación asimétrica que permitirá evitar que las presiones de los organismos internacionales estén puestas sobre la empresa telefónica mexicana.

Así, luego de tres años de amparos y declaraciones por parte de Telmex y de la COFETEL, se dio a conocer la aparición de una reglamentación especial de tipo asimétrico para controlar a Telmex. ${ }^{22}$ No obstante, sigue habiendo asuntos sobre la mesa sobre los cuales todavía no hay una resolución aparente. Internet es uno de ellos. Este es un problema que enfrenta no sólo México y la mayoría de los países prefieren no tocar el punto y dejar que el mercado regule solo la operación.

Debido a los compromisos contraidos como resultado de la firma del TLC México ha tenido que desarrollar una infraestructura informática y de telecomunicaciones a paso veloz que le ha permitido un gran avance de su infraestructura telefónica, informática y de internet. En ese sentido se puede convertir en líder en América Latina. Sin embargo, mucho del crecimiento está dándose sin planeación y al ritmo que marcan los intereses de las grandes compañías del sector.

El aparente éxito de la privatización y la derregulación en el sector de las telecomunicaciones en México, nuestro país tendrá que asumir las consecuencias de un rápido crecimiento de mercado en unos cuantos años y de una competencia feroz de las grandes compañías de telecomunicaciones por incorporar a México dentro de los mercados globales. ${ }^{23}$ Esto ha llevado a las compañías de telefonía, a una guerra sin cuartel por los consumidores y usuarios.

21. Fuente: Norman Lerner. "The Latin Experience" en Telecommunications on line. Apritl 1999 http://www.telecoms-mag.com/issues/199904/tci/latin.html

22. Ilyana Guzmán Reyes (enviada). "Lista la regulación asimétrica para Telmex. Anuncia Jorge Nicolín que se darpa a conocer en esta semana" en: El Financiero. Seción Telecomunicaciones e informática. Lunes 11 de septiembre de 2000, pág. 60

23. De acuerdo con datos de la COFETEL, nada más durante el año de 1996 se autorizó la operación de 15 compañías de servicios móbiles, satélite y paging, que representan opciones de mercado totalmente nuevas para los consumidores mexicanos. 
Las grandes compañías de hardware y software saben que el futuro del mercado está en México y en el resto de Latinoamérica y que internet apenas comienza. Según Compaq por ejemplo, el proceso de modernización del Estado y el sector educativo son dos nichos de suma importancia en los cuales la industria está enfocando todos sus esfuerzos. ${ }^{24}$.

En cuestión de unos cuantos años México tuvo que cambiar toda su infraestructura de comunicaciones para hacerse capaz de manejar la competencia tecnológica. Ello implicó la instalación de fibra óptica, enlaces de microondas y la renovación de todo el cableado existente en más de 15,000 kilómetros, con un costo aproximado de diez mil millones de dólares. No obstante, con la ayuda de compañías extranjeras como Ericsson, ahora el mercado tiene un valor de mil ochocientos millones de dólares y se espera que para el año 2001 su valor incremente a dos mil quinientos millones de dólares.

Las cifras son impresionantes, si se considera que hace tan sólo unos años México se encontraba sumergido en una crisis financiera a nivel internacional, sin embargo, basados en la predicción natural de la importancia de la información y la transmisión de datos en un mercado global cada vez más interrelacionado, ello no desalentó a los capitales extranjeros a invertir en el negocio de las telecomunicaciones.

La perspectiva es la integración de nuevos servicios de valor agregado al cúmulo de posibilidades que ahora ofrece la infraestructura instalada; de esta manera, empresas como Telmex están ahora ofreciendo servicios de voz, transmisión de datos, fax y conexión a internet, lo cual se convierte en su más importante atractivo.

Ahora, otras empresas relacionadas con la industria de las comunicaciones, como los grandes consorcios televisivos; Televisión Azteca y Televisa, han decidido entrar a la industria de las telecomunicaciones. Azteca a través de su filial Elektra, está ofreciendo servicios de telefonía celular y transmisión de datos, mientras que Televisa ha entrado fuertemente al negocio de la internet. ${ }^{25}$

Ahora bien, de acuerdo con la Unión Internacional de Telecomunicaciones (UIT), México tiene apenas una teledensidad de apenas 10 líneas telefónicas por cada 1,000 habitantes, de tal suerte que hay mucho por hacer. Las predicciones del sector es que la industria llegue a conseguir una teledensidad de 40 a 45 puntos durante los próximos 15 años. Por lo que, pese a todas las vicisitudes que la endeble situación

24. Enrique Ospina, vicepreseidente general de Compaq Latin America. Declaraciones a El Financiero en "México puede ser líder tecnológico en América Latina: Compaq", Lunes 11 de septiembre de 2000 , pág. 61

25. Roberto Aguilar, "Favorecen a Televisa expectativas en Internet" en Reforma, diciembre de 1999, p. $15 \mathrm{~A}$. 
económica o política del país pueda ofrecer, México sigue siendo una gran oportunidad de mercado para las compañías fuertes en esta industria. ${ }^{26}$

Una prueba de que este sector está siendo considerado como la gran apuesta del futuro, está en el hecho de que los grandes analistas financieros como Business Communications Company esperan que el mercado de equipo para telecomunicaciones crezca a un ritmo de un 9 por ciento anual, y llegue a aproximadamente 30 mil millones de dólares para el año $2002 .{ }^{27} \mathrm{De}$ acuerdo con esta misma fuente, México experimentará el crecimiento más acelerado del mercado latinoamericano, 10 por ciento anual que se registrará entre los años de 1999 y $2002 .^{28}$

Latin American Market for

Telecom Equipment, 1998-2000

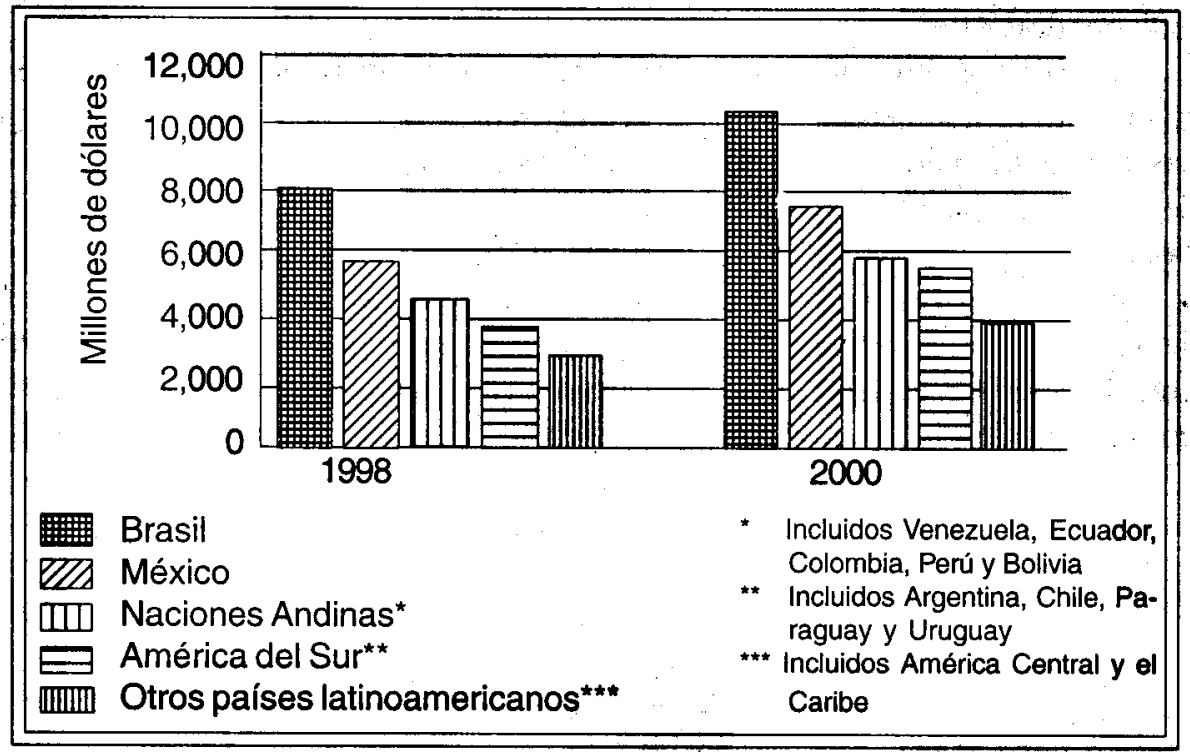

Fuente: BBC

La entrada de nuevos actores económicos, políticos y sociales al negocio de la informática y las telecomunicaciones nos presenta un panorama para México, muy diferente del que existía anteriormente cuando el país se dió a la tarea de iniciar la tarea de incorporar nuevas tecnologías para los distintos sectores prioritarios del país.

26. Fuente: Bhawani Shankar, "México in the Heat of Competition" en Telecommunications on line. May, 1988, http://www.telecoms-mag.com/issues/199805/tci/shanmexi.html

27. Business Communications Company (BCC), www.buscom.com. 26

28. Ibid. 
Actualmente, no son nada más las compañías que tradicionalmente se dedicaban al sector de la telefonía, como Teléfonos de México (Telmex) quienes están inmersos en el negocio. Empresas que nacieron dedicadas a la industria del entretenimiento como Televisión Azteca y Televisa, se encuentran en una franca y abierta competencia por entrar a formar parte del mercado de la información.

Para Telmex, la oportunidad de oro se dió con la privatización y con la promulgación de la Ley Federal de Telecomunicaciones de 1995 que, en acuerdo con el TLC, desligó a la telefonía como área exclusiva de influencia del Estado y abrió el sector a la iniciativa privada. Por otra parte, la globalización del sector le permitió a Telmex incursionar en otros mercados fuera de México, como el Sur de los Estados Unidos y Centro América para compensar la influencia de las trasnacionales que llegaron a competir en servicios de larga distancia internacional. Es claro, sin embargo, que su ventaja competitiva más clara estará no en los servicios de telefonía básica, sino en los servicios de valor agregado, fax, transmisión de datos, internet y multimedia,

\section{Actividades de expansión de Telmex}

\begin{tabular}{|c|c|}
\hline $\begin{array}{l}\text { Servicios de larga distancia al mercado hispano } \\
\text { de los Estados Unidos }\end{array}$ & 80 mdd \\
\hline $\begin{array}{l}\text { Compañía de servicios inalámbricos } \\
\text { Comm South, en Dallas }\end{array}$ & 70 millones \\
\hline $\begin{array}{l}\text { Compra de Cellular Communications of Puerto } \\
\text { Rico, conjuntamente con SBC Communications }\end{array}$ & $\begin{array}{l}464 \text { mdd en efectivo } \\
\text { más deuda de } 350 \text { mdd }\end{array}$ \\
\hline $\begin{array}{l}\text { Participación en CDNow, mayorista de venta } \\
\text { de CD's y servicios de intemet }\end{array}$ & $17 \mathrm{mil} 500 \mathrm{mdd}$ \\
\hline $\begin{array}{l}\text { Convenio con la empresa de redes de fibra óptica IPO } \\
\text { of Williams Communications Group, de Tulsa, Oklahoma }\end{array}$ & 100 mdd \\
\hline Participación accionaria en Grupo Televisa* & 350 mdd \\
\hline $\begin{array}{l}\text { Participación en Topp Telecom, proveedor } \\
\text { de servicios inalámbricos en Miami }\end{array}$ & $57 \mathrm{mil} 500 \mathrm{mdd}$ \\
\hline Acciones transferibles & 100 mdd \\
\hline $\begin{array}{l}\text { Compañía Telefónica Guatemalteca. } \\
\text { Contrato de Administración }\end{array}$ & $\begin{array}{l}\text { Compra del } 49 \text { por } \\
\text { ciento no disponible }\end{array}$ \\
\hline $\begin{array}{l}\text { Participación en la privatización la Compañía } \\
\text { Nicaraguense de Telefonía }\end{array}$ & $\begin{array}{l}\text { Retiro de la } \\
\text { participación }\end{array}$ \\
\hline $\begin{array}{l}\text { Participación en la privatización de la Compañía } \\
\text { Telefónica de El Salvador }\end{array}$ & $\begin{array}{l}\text { Retiro de la } \\
\text { participación }\end{array}$ \\
\hline
\end{tabular}

*A través del Grupo Carso/Inbursa, la empresa de por sí ya poseia el 49 por ciento de Cablevision, el operador de cable más importante de México, perteneciente a Grupo Televisa. Fuente: Wall Street Journal, rRanscomm, Inc. 
para lo cual Telmex ha buscado alianzas estratégicas con otras compañías internacionales. Así por ejemplo, para atender los mercados emergentes del Sur de Estados Unidos y Centro América, Telmex ha unido esfuerzos con Sprint de la siguiente manera:

Es muy posible además, que las incursiones de Telmex no queden ahí, ya que el consorcio está esperando la publicación de reglas precisas para la industria del entretenimiento, que le permita ampliar su participación hacia la industria de la televisión de alta definición, la televisión directa al hogar e internet.

En el caso de internet por ejemplo, el gobierno mexicano no ha establecido ninguna regulación precisa para los servicios de interconexión para los proveedores nacionales, más allá de los límites que imponen los servicios de larga distancia. En cuanto a los proveedores extranjeros de servicios de interconexión, el propio gobierno norteamericano está dejando libre el sector con el propósito de promover su crecimiento y desarrollo, por lo que es muy posible que por el momento, por lo menos mientras la adopción y el consumo se generalizan para garantizar un mercado más estable, no se modifiquen las disposiciones a este respecto. Por lo pronto, proveedores como CompuServe e Infosel, Internet de México y DataNet son parte ya de la compañía norteamericana Psinet. No hay que olvidar, además, que otras industrias relacionadas tales como la televisión por cable, y otros servicios de comunicación inalámbrica buscarán eventualmente aprovechar los servicios de interconexión de datos proporcionados por las redes de internet.

Por supuesto que todos estos movimientos en el sector implican cambios y transformaciones importantes en la forma en la que se comportarán los mercados en los próximos años: se hará cada vez más importante la necesidad de un marco regulatorio específico que norme la actividad de todas las industrias relacionadas. La competencia se tornará cada vez más fuerte y despiadada.

Las negociaciones que originalmente fueron establecidas a nivel de Estados nacionales entre los Estados Unidos y Canadá, con sus correspondientes transformaciones en los marcos regulatorios y en las políticas estatales, se transformará cada vez más en negociaciones internas dentro de la industria y en compras hostiles de compañías grandes que querrán atraer hacia sí los mercados más atractivos. Los servicios de telecomunicaciones (telefonía, e informática) que tradicionalmente se ubicaban en la industria, por su vinculación con la industria del entretenimiento, requerirán de mecanismos de interconexión que permitan la transmisión de datos a mayor velocidad y por lo tanto requerirán tecnología de transmisión de banda ancha.

Dada la tendencia de las empresas del sector de diversificarse e intervenir en varios mercados fuera de su ámbito original de influencia, en un principio la conver- 
gencia de mercados implicará una mayor oferta de productos, pero no necesariamente una mayor eficiencia en los servicios. ${ }^{29}$

Por supuesto, una ventaja adicional a los servicios existentes actualmente, se encuentra en la utilización y el aprovechamiento de la infraestructura satelital. Bien claro ha quedado ya, a raíz de las grandes fallas técnicas de los satélites que bañan el territorio mexicano, que se registraron en abril y mayo de 1999, y la desconexión definitiva del Solidaridad I en agosto del año 2000.

El Estado mexicano había tomado ya, como consecuencia de nuestros nexos con el bloque económico de América del Norte y la firma del TLC, la decisión de privatizar la operación de los satélites mexicanos. Ello le dio impulso a la industria satelital y trajo dinero fresco al negocio de la transmsión de datos. Sin embargo, las empresas privadas que adquirieron la concesión para la operación de los servicios estaban adquiriendo también una infraestructura caduca, pero sobre la que pendía gran parte de la actividad económica del país.

Durante las fallas técnicas de 1999, la banca tomó la decisión de cambiar sus operaciones del Satélite Solidaridad I al Solidaridad II y a la red digital de Telmex. Ello incrementó la importancia de Telmex, ya que esta empresa prácticamente cubrió el 100 por ciento de las operaciones bancarias del país. Sin embargo, en agosto del año 2000, cuando la infraestructura de operaciones del Solidaridad I volvió a fallar se quedaron fuera temporalmente el 33 por ciento de los usuarios, entre ellos 1 millón de niños y jóvenes que recibían clases vía satélite. Satmex, la empresa privada operadora de los servicios del Solidaridad I tuvo que migrar sus servicios al Solidaridad II, y a los satélites Satmex V, TelstarV, VI y VII propiedad de Loral Space \& Communications. ${ }^{30}$

Desde la privatización de los satelites, y en particular de Satmex, se ha incorporado la participación de compañias como Loral Space and Communications, quien realizó una inversión inicial de 688 millones de dólares, lo cual facilitará la incorporación de servicios integrados punto a punto y punto multipunto de datos, vídeo y otros a través de la flota geoestacionaria de satélites Loral-Skynet. Esta infraestructura reemplazará a la vieja guardia de satélites Morelos y de la serie Telstar IV, v, vi y vil. A lo anterior, hay que agregar la participación de Huges Communications a través de la compra de Panamsat. Esta compañía incorpora los servicios de Hughes Galaxy con toda una gama de posibilidades de interconexión y servicios digitales.

29. Fuente: Normar Lerner. “A Bright Market Matures" Ibid, en: http://www.telecoms-mag.com/issues/ 199910/tci/mexico.html

30. "Podría Satmex perder varios clientes" Reforma. Martes 29 de agosto del 2000. en: http:// www.reforma.com/negocios_y_dinero/artículo/030198/ 
Los compromisos de México frente a la Unión Europea, además de los ya contraídos dentro del TLC, obligan a nuestro país a desarrollar un sistema de interconexión que permita cumplir nuestras obligaciones comerciales dentro de organismos como la Organización Mundial de Comercio que coloca, por primera vez a los acuerdos de comercio multilaterales no sólo básicos sino también a los de servicios de valor agregado, dentro de los cuales se encuentran, evidentemente, los servicios de telecomunicaciones.

De los más de 130 miembros de la OMC, 31 son gobiernos latinoamericanos que caen dentro de este esquema. Ello incluye compromisos de interconexión, obligación universal de proporcionar el servicio y alocación para uso de espectros de banda. ${ }^{31}$ México se ha abocado, a través de cOFETEL, a la liberalización del sector, a la licitación pública para el otorgamiento de licencias de servicios de telecomunicaciones, a la definición de áreas de influencia y a resolver el problema de los servicios de interconexión. ${ }^{32}$.

Desde luego, a través de este recorrido es factible observar varios aspectos que resultan de interés en el desarrollo de las nuevas tecnologías para un país y en su desenvolvimiento y utilización dentro de políticas específicas: Por lo pronto es factible observar un repliegue por parte de la figura del Estado en lo que se refiere a la administración del sector de las telecomunicaciones, la informática, y en general las nuevas tecnologías; por otra parte, es posible percibir un viraje de la política de utilización de nuevas tecnologías, televisión, satélites, computación e informática, que se ha desplazado de los sectores públicos y principalmente de su utilización con fines educativos o industriales, hacia los sectores comerciales y de entretenimiento; y finalmente encontramos la incorporación de actores no solamente económicos y sociales, sino también políticos, ajenos a la mecánica nacional y que responden claramente a los compromisos signados por nuestro país en el terreno internacional con otros bloques y organismos económicos.

Como es factible observar, la infraestructura de telecomunicaciones, y en general la utilización de nuevas tecnologías en nuestro país, ha experimentado un crecimiento exponencial en los últimos años, lo cual coincide abiertamente con los movimientos de apertura comercial a nivel internacional. Mucho de lo que sucede actualmente en México es reflejo de todo ámbito latinoamericano en el sector de las comunicaciones, pero además, refleja la tendencia global de los países en términos

31. World Trade Organization, "The New WTO Agreement: Opportunities and Challenges", Telecommunications, September 1997.

32. Fuente: Normar Lerner: "Telecom Privatization in Latin America: Trends in Competition \& Regulation" en Telecommunications on line. February, 1998, en http://www.telecommag.com/issues/ 199802/tcs/lerner.html 
de indicadores para el desarrollo. La pregunta es, ante este nuevo esquema de articulación de cosas, ¿cuál será el desarrollo de la política social, educativa y cultural que espera a México, y en general al resto de los países latinoamericanos?

\section{Conclusiones}

A lo largo de este trabajo hemos presentado tres momentos distintivos en el desarrollo de las nuevas tecnologías en México. El primero, en el que las nuevas tecnologías fueron adoptadas con propósitos educativos, y en el cual unas cuantas instituciones, primordialmente de origen gubernamental se hicieron cargo del proceso de introducción de las innovaciones tecnológicas informacionales; un segundo momento en el cual el desarrollo de estas nuevas tecnologías fue visto como un prerrequisito para la apertura informática y económica del país, y un tercer momento, en al cual fueron invitados todos los demás actores del proceso económico y político del país. Este es precisamente el momento en el que se encuentra nuestro país, en el cual el sector de las telecomunicaciones y de la informática se ha convertido en la nueva actividad prioritaria de la economía, y que muy probablemente desarrollará todo un nuevo esquema de articulación, social, educativa, política y cultural.

Ese nuevo esquema de articulación, descansa sobre una base múltiple: un primer eje es el tope de crecimiento de los mercados. El crecimiento exponecial de los mercados, que si bien está previsto, permanecerá por lo menos durante la primera década del próximo milenio, muy probablemente también decaiga al alcanzar un tope de utilidad para las compañías participantes.

Un tercer eje, sin embargo, y quizás el primero en importancia, será la fusión, ya irreversible entre los servicios de información y entretenimiento, lo cual dejará por supuesto a la educación en un papel o situación de conflicto, ya que a menos que sea negocio, y se encuentre vinculada directamente con una opción comercial de entretenimiento, la educación con tecnología informática de punta, tenderá a ser impulsada por compañías privadas, dejando exclusivamente la función de coordinación a un sector público cada vez más empobrecido y amilanado.

Por último, y ese es justamente el talón de Aquiles de toda la utopía, la interconexión y las posibilidades de comunicación que nos brindarán las nuevas tecnologías en el siglo XXI, no harán sino llevar al núcleo de todas nuestras problemáticas, el desarrollo de la industria, el comercio, la educación, la información y el entretenimiento, junto con la inconsistencia de una infraestructura que permite una articulación nacional e internacional pero que nos vincula como entidades, como países y 
como individuos a una información vacía que poco o nada nos brinde en términos de definirnos como sociedades ricas en términos identitarios.

\section{Bibliografía}

Aguilar, Roberto, "Favorecen a Televisa expectativas en Internet", en Reforma, Miércoles 15 de diciembre de 1999, p. 15a.

Aguilar, Roberto, "Plantea Eu ultimátum en telecomunicaciones. Afirma que México protege un monopolio televisivo", en Reforma, Miércoles 26 de julio de 2000, Sección A, p. 1.

Business Communications Company Inc. (BCC) (información periódica), en www.buscom.com.

Davis, Charles, "Algunos desafíos para el desarrollo del comercio electrónico en la América Latina de habla hispana", en http://ute.edu.ec/ mjativa/ce/ tendencias.html, marzo 1998.

Fernández Hernández, Patricia, "Experiencias institucionales de educación a distancia", IV Encuentro-Taller Internacional de Educación Continua del Programa Columbus, septiembre, 1988.

Gall, Ruth et al., Las actividades espaciales en México: Un revisión crítica. Fondo de Cultura Económica/sEP/CONACYT, 1986.

García Jiménez, Jesús, Televisión educativa para América Latina, Porrúa. México, 1970.

Grupo Reforma, “Podría Satmex perder varios clientes”, Reforma, Martes 29 de agosto del 2000, en: http://www.reforma.com/negocios_y_dinero/artículo/030198. Guadarrama, José de Jesús, "México puede ser líder tecnológico en América Latina: Compaq. Propone la modernización de infraestructura tecnológica", en $E l$ Financiero. Lunes 11 de septiembre de 2000., p. 61.

Guzmán Reyes, Ilyana, "Lista la regulación asimétrica para Telmex. Anuncia Jorge Nicolín que se dará a conocer en esta semana", en El Financiero. Lunes 11 de septiembre de 2000, p. 60.

Headcount. Com. servicio de International. Com., en: http://www.headcount.com/ count/datafind.htm?id=153\&choice $=$ country.

Innis, Harold, Communication and Empire, Oxford, 1950.

International Data Corporation, Junio de 1999.

Internet Domain Service, hhtp://www.nw.com.

Kuhlmann, Federico, Antonio Alonso C. y Alfredo Mateos, Comunicaciones: Pasado y Futuros, Fondo de Cultura Económica, México, 1989. 
Nieto, Dulce, "Perfil de la educación en México", Secretaría de Educación Pública, http://www.sep.gob.mx/sen/perfil/p7_1.htm.

Norman, Lerner, "Telecom Privatization in Latin America: Trends in Competition \& Regulation", en Telecommunications on line. February, 1998, en http:// www.telecoms-mag.com/issues/199802/tcs/lerner.html.

Norman Lerner, "The Latin Experience", en Telecommunications on line, April 1999, http://www.telecoms-mag.com/issues/199904/tci/latin.html.

Lerner, Norman, "A bright Market Matures" en Telecommunications on line. October, 1999, http://www.telecoms-mag.com/issues/199910/tci/mexico.html.

Santoyo, Ramón V., "Internet, Banca, y Comercio Electrónico", Grupo Telelink, en http://www.telelink.com.mx/docs/tecnofin/sld001.htm, 1997.

Secretaría de Hacienda y Crédito Público de México, "Programa de Desarrollo Informático", INEGI, México, 1980.

Shankar, Bhawani, "México in the Heat of Competition", en Telecommunications on line, 1988, http://www.telecoms-mag.com/issues/199805/tci/shanmexi.html.

Teléfonos de México. "Orígenes de la Telefonía", en http://www.telmex.com.mx/ estoes/historia/historia_frame.htm.

World Trade Organization, "The New wTo Agreement: Opportunities and Challenges", Telecommunications, September, 1997. 